\title{
Diagnostic Value of Portal Venous Pulsatility Index in Patients with High-Risk Nonalcoholic Fatty Liver Disease
}

\author{
Nadia Haleem Hamed*, Manal Farouk Al Tohamy, Amr Osama Khalil, Mohammed Ibrahim Amin \\ Department of Radiodiagnosis, Faculty of Medicine, Zagazig University, Egypt \\ *Corresponding author: Nadia Haleem Hamed, Email: nadiafadda1@ gmail.com
}

\begin{abstract}
Background: As commonest form among liver diseases, nonalcoholic fatty liver disease (NAFLD) presents no symptoms. It is possible to use regular ultrasound scanning, which are always available in medical care centers everywhere, to quickly and quantitatively assess portal vein pulsatility. Objective: To evaluate the value of the portal venous pulsatility index for noninvasively diagnosing nonalcoholic fatty liver disease who are at high risk.

Patients and Methods: The trial was a comparative cross-sectional trial involving 145 NAFLD patients, conducted in Radiodiagnosis Department, Faculty of Medicine, Zagazig University Hospitals. Abdominal ultrasound fibroscan, and Doppler US examinations of portal venous system were done to all patients.

Results: Basal lab features, triphasic right hepatic vein flow pattern, and Doppler flow characteristics differed significantly between the two groups. Except for the BARD score, there was a high statistically significant difference between the two groups for venous pulsatility index values as well as all clinical risk scores. VPI, NAFLD-FS, and FIB-4 odds ratios all showed high significant differences between the two groups. Optimism-Corrected ROC AUC of VPI +NAFLD FS was 0.89, VPI + FIB-4 was 0.90, VPI+BARD score was 0.86 and VPI + APRI was 0.85. There was a high significant difference regarding VPI +NAFLD FS \& VPI + FIB-4.
\end{abstract}

Conclusion: High-risk NAFLD can be predicted using VPI and this could improve the effectiveness of frequently used clinical predictor tools.

Keywords: Nonalcoholic fatty liver disease, Venous pulsatility index.

\section{INTRODUCTION}

As long as alcohol consumption isn't excessive, it's possible to develop NAFLD, which is marked by fat collection at parenchymal liver (known medically as hepatic steatosis). Because of its prevalence and lack of symptoms, NAFLD is the world's most common liver conditions (1). Approximately $25.2 \%$ of people worldwide have nonalcoholic fatty liver disease, and that number rises to about $31.8 \%$ among Middle East population. About 33\% of Americans are affected, associated with metabolic syndrome's comorbidities, such as being diabetic, overweight or having high cholesterol. In diabetics, NAFLD affects roughly $60 \%$ of those who have it ${ }^{(2)}$, whereas in obese individuals, this number is increasing to 90 percent ${ }^{(3)}$. As far as nonalcoholic fatty liver disease develops, it encompasses several different types of liver damage, ranging from mild simple steatosis to severe steatohepatitis [nonalcoholic steatohepatitis (NASH)], and cirrhosis as well liver fibrosis ${ }^{(4)}$.

There are five stages of fibrosis in NAFLD, according to the NASH Clinical Research Network (NASH CRN) classification scheme ${ }^{(5)}$ : Fibrosis types include F Zero (where there is no fibrosis), F One (portal fibrosis without septa), F2 (portal fibrosis with low number of septa), F3 (central vein bridging septa), and F4 (cirrhosis) ${ }^{(5)}$. When liver fibrosis progresses to stage F2 or higher, the long-term mortality and morbidity accompanied with the liver are significantly increased ${ }^{(\mathbf{5})}$. As a result, expression of high-risk NAFLD refers to people whose disease has progressed beyond the F2 stage. Monitoring for high-risk fatty liver disease is critical in the treatment of NAFLD since it identifies the patients who mostly in need of treatment and study ${ }^{(6)}$.

Liver biopsy is the gold standard for diagnosing liver fibrosis. As a result of the procedure's invasiveness, high cost, and danger of uncommon but serious consequences and sampling errors, it isn't used as a screening tool ${ }^{(7)}$. There are therefore several noninvasive methods of staging liver fibrosis, like serum biomarkers, fibroscan, as well as clinical-laboratory decision aids, that have been developed, such as BARD score (body mass index), fibrosis-4 index (FIB-4), the NAFLD fibrosis score (NAFLD FS), AST to platelet ratio index (APRI), and aspartate aminotransferase to alanine aminotransferase ratio (AST/ALT). However, these methods have drawbacks when employed in clinical practice ${ }^{(8)}$.

Now, the most promising noninvasive way to diagnose liver fibrosis is transient hepatic elastography (THE). Rapidity and safety are the primary benefits of this procedure ${ }^{(9)}$. In individuals with NAFLD, transient hepatic elastography outperformed APRI, FIB4 and the NAFLD fibrosis score when compared to the gold standard, introducing the best performance for diagnosing and excluding advanced fibrosis ${ }^{(\mathbf{1 0})}$.

As an ultrasound biomarker for liver fibrosis, one of the most commonly used ones is the portal vein pulsatility index (VPI), which is a measurement of portal vein duplex Doppler assessment and a quantitative measure of venous pulsatility that is estimated by (Vmax - Vmin) / Vmax, the maximum pulsed-wave Doppler ultrasonography estimate of blood velocity in the portal vein is Vmax, whereas the minimum is Vmin. It is 
possible to use regular ultrasound scanners, which are always available in medical care centres everywhere, to quickly and quantitatively assess portal vein pulsatility (11).

Our study's goal is to determine noninvasively portal venous pulsatility index utilization to identify patients with nonalcoholic fatty liver disease at high risk.

\section{PATIENTS AND METHODS}

From June 2020 to October 2021, we conducted a comparative cross-sectional trial on 145 patients with NAFLD at the Radiodiagnosis Department, Faculty of Medicine, Zagazig University Hospitals.

\section{Ethical consent:}

An approval of the study was obtained from Zagazig University Academic and Ethical Committee. Every patient signed an informed written consent for acceptance of the study. This work has been carried out in accordance with The Code of Ethics of the World Medical Association (Declaration of Helsinki) for studies involving humans.

Inclusion criteria: Patients aged group from 20 to 65 years from both sexes with nonalcoholic fatty liver disease.

Exclusion criteria: Non-cooperative patients like patients refusing full investigations and or not completing follow-up. There was no other exclusion to any patient as the Doppler US examination is almost safe. Non-ionizing radiation is used, therefore there are no X-ray-like dangers like there are with other imaging methods.

On the basis of the seriousness of their conditions, patients were classified into two groups: A total of 91 participants were assigned to the low-risk Group 1 (F0F1) study.

Number of individuals in Group 2 (those at higher risk): 54

\section{All patients were subjected to the following:}

1) Taking complete history.

2) A complete medical evaluation.

3) Imaging including: abdominal ultrasound fibroscan, and Doppler US examinations of portal venous system

- Patients examined clinically who had undergone liver shear wave elastography, which was implemented on a convex probe (C5-1) of PHILIPS IU22 ${ }^{\mathrm{TM}}$, diagnosed as Nonalcoholic Fatty Liver disease patients. There were four grades of liver fibrosis, each with a score between 0 and 4, which correspond to the Metavir histological index for classifying liver fibrosis.

4) The PHILIPS IU22TM device's fibrosis stage cutoff values were as follows, taken from the device's manual and default settings: (1) F0-F1 mild fibrosis (three to seven KPs). (2) 7-12 KPs of moderate fibrosis (F2). (3) Severe fibrosis (F3), measuring 12-21 KPs. (4) More than $21 \mathrm{KPs}$ of cirrhosis (F4).

\section{Doppler ultrasound imaging:}

- Licensed medical sonographers using Canon Applio I500TM (GE Healthcare) ultrasound scanners performed ultrasound studies within the designated period.

- All patients were subjected to a pulsed-wave Doppler ultrasonography examination of the portal vein following a 4-hour fast, as per usual procedure.

- Imaging was done in a supine position with a breath-hold at the conclusion of a typical expiration.

- The PACS was used to retrieve ultrasound imaging data.

- Estimated maximum and lowest portal venous velocities can be measured using the spectral pictures. VPI (Vmax-Vmin)/Vmax was then calculated using these measurements.

Finalizing the work: Dividing the patients into 2 groups low risk group (group A) \& high risk group (group B).

\section{Statistical analysis:}

The collected data were placed onto a computer and ran through the Statistical Package for Social Sciences, version 28 (SPSS). Tables and graphs were utilized to convey the results. Analysis of variables' distribution characteristics and homogeneity of variance was carried out using the Shapiro-Wilk test. The quantitative data was presented in the form of mean, median, standard deviation, and confidence intervals. The frequency and percentage of qualitative data were employed in the presentation of the material. The student's t test (T) and Mann-Whitney test (MW) were used to assess quantitative independent data as required. Pearson Chi-Square Test and the Chi-Square for Linear Trend were used to assess qualitatively independent data. In order to be declared statistically significant, $\mathrm{P}$ value has to be equal or less than 0.05 .

\section{RESULTS}

Group 1 (Low risk group): It included 90 subjects with ages ranging between 20 and 65 years and mean age was $43.12 \pm 10.5$. Males were $49.5 \%$ and females were $50.5 \%, 25 \%$ of this group were diabetics. Weight ranged between 65 and 120 kilograms with a mean of $89.62 \pm 16.85$ years. Height ranged between 1.3 and $1.9 \mathrm{~m}$ with a mean of $1.65 \mathrm{~m}$ \pm 0.3 . BMI mean was $30.5 \pm 6.3$.

Group 2 (High risk group): It included 54 subjects with mean age of $42.6 \pm 9.6$ years ranging between 20 and 65 years. Males were $53.7 \%$ and females were $46.3 \%, 29.63 \%$ of this group were 
diabetics. Weight ranged between 62 and 118 kilograms with mean of $90.5 \pm 14.3$ years. Height ranged between 1.21 and $1.95 \mathrm{~m}$ with mean of 1.65 $\mathrm{m} \pm 0.3$. BMI mean was $31.1 \pm 5.5$ as shown in table (1). Regarding demographic characteristics, no significant difference was found between the two groups.

(Table 2) showed that in low risk group, venous pulsatility index mean reached 0.28 ranging from 0.21 to 0.36 . NAFLD fibrosis score was -2.32 ranging between -2.05 and -1.1 . FIB-4 mean was 1.13 ranging between 0.74 and 1.63. BARD score was 2 ranging between 1 and 3. APRI was 0.42 ranging between 0.31 and 0.65 . In high risk group, venous pulsatility index mean reached 0.18 ranging from 0.13 to 0.21 . NAFLD fibrosis score was -0.71 ranging between -1.69 and -0.13 . FIB-4 mean was 2.11 ranging between 1.43 and 2.89. BARD score was 3 ranging between 1 and 3. APRI was 0.65 ranging between 0.41 and 1.03. Both groups had substantial variations in terms of baseline lab features and Doppler flow characteristics ( $\mathrm{p}$ value $<0.05$, 0.05 , and 0.001 respectively).

With the exception of the BARD score, there were high statistically significant differences between the two groups among all clinical risk scores and venous pulsatility index values (table 3 ).

(Table 4) showed that there was a high significant difference between the two groups regarding VPI, NAFLD-FS and FIB-4 Odds ratios. There was a significant difference regarding APRI. However, there was no significant difference regarding BARD score.

(Table 5) showed that Optimism-Corrected ROC AUC of VPI +NAFLD FS was $0.89(0.80$ 0.96), VPI + FIB-4 was 0.90 (0.85-0.97), VPI+BARD score was $0.86(0.77-0.93)$ and VPI + APRI was $0.85(0.76-0.94)$. There was a high significant difference regarding VPI +NAFLD FS \& VPI + FIB-4.

Table (1): Demographic characteristics

\begin{tabular}{|c|c|c|c|}
\hline Variable & $\begin{array}{c}\text { Low-Risk NAFLD } \\
(\mathrm{n}=91)\end{array}$ & $\begin{array}{c}\text { High-Risk NAFLD } \\
(n=54)\end{array}$ & P-value \\
\hline Age & $43.12 \pm 10.5$ & $42.6 \pm 9.62$ & \multirow{8}{*}{$>0.05$} \\
\hline Sex & & & \\
\hline Male & $45(49.5 \%)$ & $29(53.7 \%)$ & \\
\hline Female & $46(50.5 \%)$ & $25(46.3 \%)$ & \\
\hline Diabetes & $23(25.27 \%)$ & $16(29.63 \%)$ & \\
\hline Weight $(\mathrm{Kg})$ & $89.62 \pm 16.85$ & $90.5 \pm 14.3$ & \\
\hline Height (m) & $1.65 \pm 0.3$ & $1.71 \pm 0.25$ & \\
\hline BMI (Kg/m2) & $30.5 \pm 6.3$ & $31.1 \pm 5.5$ & \\
\hline
\end{tabular}

Table (2): Basal lab. characteristics, Doppler flow characteristics of studied group

\begin{tabular}{|c|c|c|c|}
\hline Variable & Low-Risk NAFLD & High-Risk NAFLD & \multirow{2}{*}{ P-value } \\
\cline { 1 - 3 } Platelet count $(\times 109 / \mathrm{L})$ & $230.8 \pm 9.5$ & $250.48 \pm 8.1$ & \multirow{2}{*}{$>0.05$} \\
\cline { 1 - 3 } Albumin $(\mathrm{g} / \mathrm{dL})$ & $4.6 \pm 0.5$ & $4.5 \pm 0.4$ & \\
\hline AST $(\mathrm{U} / \mathrm{L})$ & $46.9 \pm 7.2$ & $59.9 \pm 8.9$ & \\
\hline ALT $(\mathrm{U} / \mathrm{L})$ & $67.5 \pm 9.6$ & $66.4 \pm 12.3$ & \\
\hline & Doppler flow characteristics & \multirow{2}{*}{$<0.001$} \\
\hline VPI & $0.32 \pm 1.25$ & $0.20 \pm 2.61$ & \\
\hline MFV $(\mathrm{cm} / \mathrm{sec})$ & $14.9 \pm 0.63$ & $11.8 \pm 1.26$ & \\
\hline Vmax $(\mathrm{cm} / \mathrm{sec})$ & $42.1 \pm 0.89$ & $29.9 \pm 4.41$ & \\
\hline Vmin $(\mathrm{cm} / \mathrm{sec})$ & $27.9 \pm 0.61$ & $21.8 \pm 3.1$ & \\
\hline
\end{tabular}

$\mathrm{ALT}=$ alanine aminotransferase. $\mathrm{AST}=$ aspartate aminotransferase,

Table (3): Assessments of clinical risk factors and the venous pulsatility index nonalcoholic fatty liver disease (NAFLD) Pathologic Diagnoses are categorized into low- and high-risk groups

\begin{tabular}{|c|c|c|c|}
\hline Scoring System & Low-Risk NAFLD $(\mathbf{n}=91)$ & High-Risk NAFLD (n=54) & P-value a \\
\hline Venous pulsatility index & $0.28(0.21,0.36)$ & $0.18(0.13,0.21)$ & $<0.001$ \\
\hline NAFLD fibrosis score & $-2.32(-2.05,-1.1)$ & $-0.71(-1.69,-0.13)$ & $<0.001$ \\
\hline FIB-4 & $1.13(0.74,1.63)$ & $2.11(1.43,2.89)$ & $<0.001$ \\
\hline BARD score & $2.00(1.00,3.00)$ & $3.00(1.00,3.00)$ & 0.053 \\
\hline APRI & $0.42(0.31,0.65)$ & $0.65(0.41,1.03)$ & $<0.001$ \\
\hline
\end{tabular}

FIB-4 = fibrosis-4 index, aspartate aminotransferase (AST)-to-alanine aminotransferase ratio, diabetes mellitus;

APRI = AST-to-platelet ratio index. a comparison of high- and low-risk NAFLD. Computed by Wilcoxon test BARD = body mass index (weight in kilograms divided by the square of height in meters). 
Table (4): Logistic Univariate Analyzing Regression to determine the diagnostic performance of (NAFLD)

\begin{tabular}{|c|c|c|c|}
\hline Model & Odds ratio & \multirow{2}{*}{ P- Value } & $\begin{array}{c}\text { Optimism-Corrected } \\
\text { ROC AUC }\end{array}$ \\
\hline VPI & $0.84(0.81-0.93)$ & & $0.86(0.75-0.92)$ \\
\cline { 1 - 2 } & $1.84(1.43-2.52)$ & $<0.001$ & $0.72(0.62-0.84)$ \\
\hline FAFLD-FS & $4.84(2.51-9.48)$ & & $0.86(0.73-0.91)$ \\
\hline BARD score & $1.41(0.98-1.98)$ & 0.063 & $0.62(0.44-0.74)$ \\
\hline APRI & $3.73(1.35-10.48)$ & 0.01 & $0.72(0.60-0.83)$ \\
\hline
\end{tabular}

VPI = venous pulsatility index, BARD = body mass index (weight in kilograms divided by the square of height in meters), FS = fibrosis score, FIB-4 = fibrosis-4 index, diabetes mellitus; APRI = AST-to-platelet ratio index. a Associated with a 1-unit change in either NAFLD-FS, FIB-4, BARD score, or APRI and a 0.1-unit change in VPI.

Table (5): Logistic multivariable High-Risk Nonalcoholic Fatty Liver Disease prediction using regression analysis to quantify diagnostic performance of scores

\begin{tabular}{|c|c|c|c|}
\hline Model & Odds ratioa & P- Value & $\begin{array}{c}\text { Optimism-Corrected } \\
\text { ROC AUC }\end{array}$ \\
\hline VPI +NAFLD FS & $0.85(0.79-0.92)$ & $<0.001$ & $0.89(0.80-0.96)$ \\
VPI & $1.92(1.29-2.82)$ & 0.001 & \\
NAFLD FS & $0.88(0.82-0.95)$ & $<0.001$ & $0.90(0.85-0.97)$ \\
VPI + FIB-4 & $4.16(2.03-8.62)$ & $<0.001$ & \\
VPI & $0.82(0.76-0.93)$ & $<0.001$ & $0.86(0.77-0.93)$ \\
FIB-4 & $1.59(1.0 .6-2.41)$ & 0.032 & \\
VPI+BARD score & & & $0.85(0.76-0.94)$ \\
VPI & $0.89(0.82-0.96)$ & $<0.001$ & \\
BARD score & $2.15(0.82-5.76)$ & 0.134 & \\
VPI + APRI & &
\end{tabular}

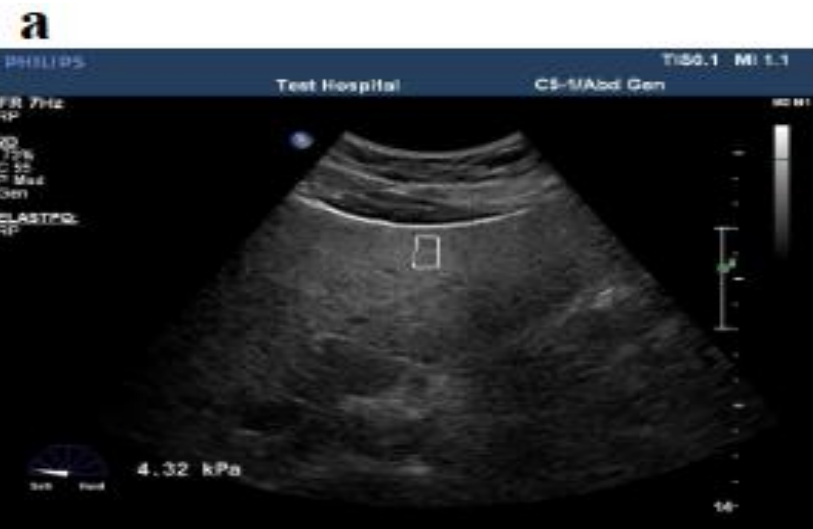

C

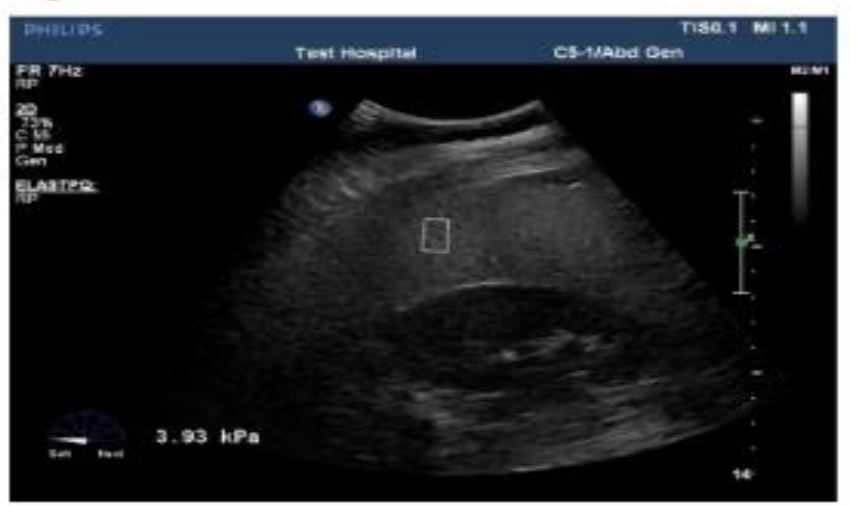

b

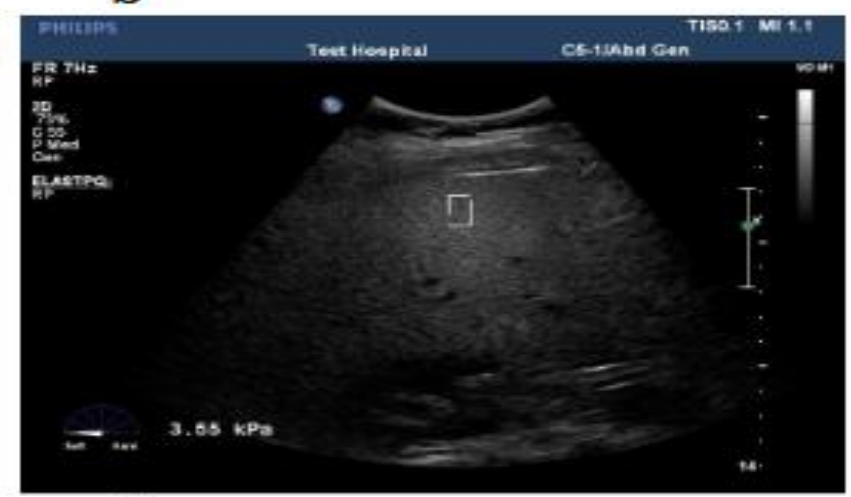

d

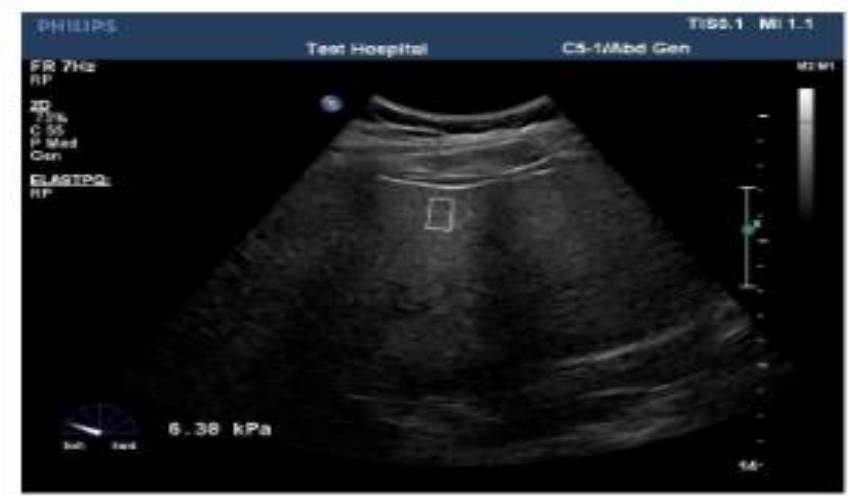

Figures: $(a, b, c, d)$ : fibroscan images through the liver at different levels and segments show stiffness average of ( $4.52 \mathrm{kPa}$ ) so patient's stage is ( $F O-F I)$. 


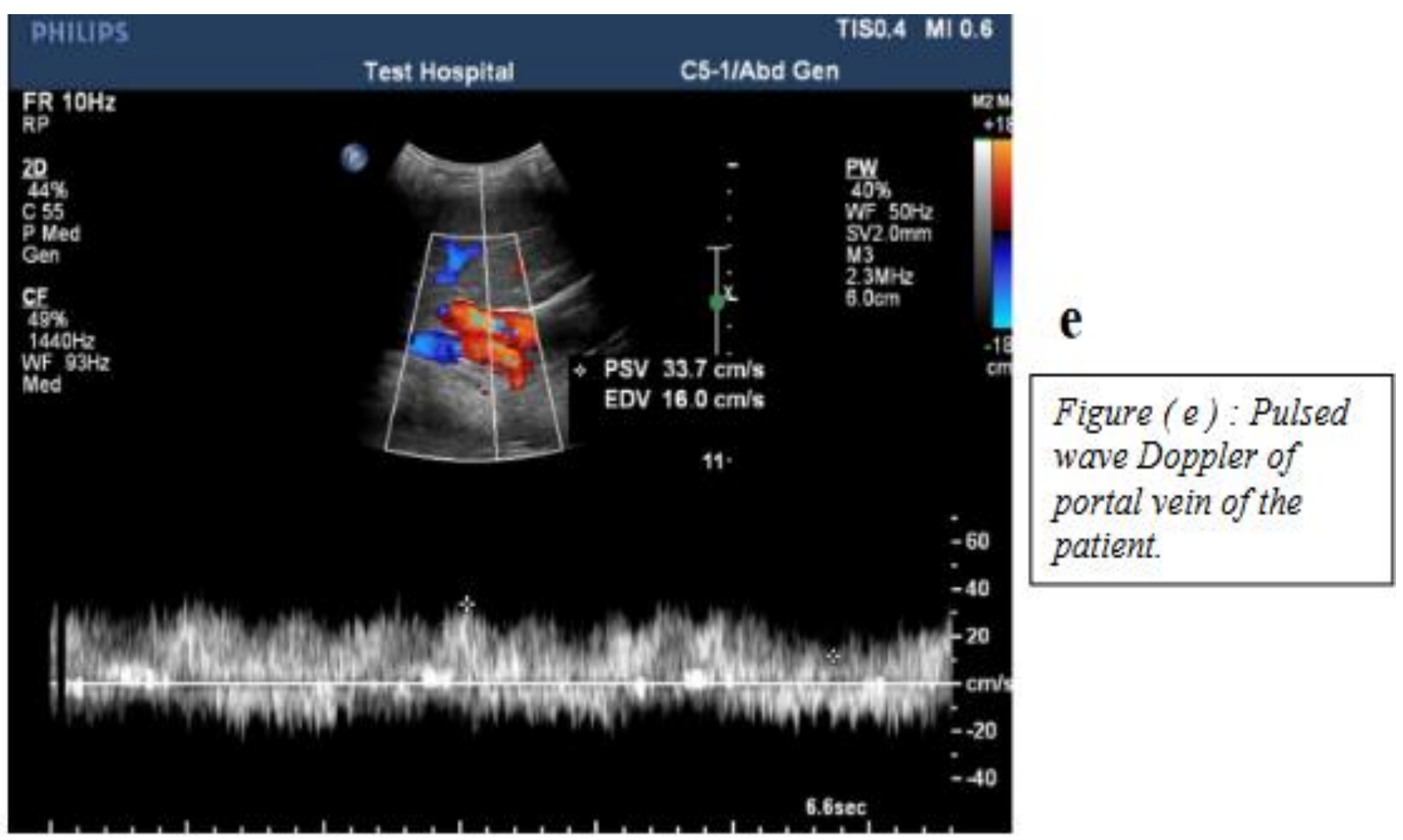

Figure (1): Male patient, 48 years old, with no history of alcohol ingestion, known asymptomatic non-alcoholic fatty liver diseased patient diagnosed clinically and by fibroscan in the Hepatology Outpatient Clinic, with no history of DM or HTN. Fibroscan of the liver done for the patient at different levels and segments showing stiffness average of $4.52 \mathrm{kPa}$. So, patient confirmed to be stage (F0-F1). Pulsed wave Doppler of Portal vein done revealed: Maximum velocity $(\mathrm{Vmax})=\mathrm{PSV}=33.7 \mathrm{~cm} / \mathrm{s}$, minimum velocity $(\mathrm{Vmin})=\mathrm{EDV}=16.0 \mathrm{~cm} / \mathrm{s}$ and pulsatility index $=\frac{\text { Vmax }- \text { Vmin }}{V \max }=0.53$

\section{DISCUSSION}

Nonalcoholic fatty liver disease is the most common cause of abnormalities in liver function testing, according to hepatology researches. About $15 \%$ to $25 \%$ of the world's population suffers from NAFLD, which is characterized by hepatocyte lipid accumulation without a prior history of heavy alcohol consumption (12). An investigation into the noninvasive diagnostic utility of a nonalcoholic fatty liver disease index known as the Portal Venous Pulsatility Index was the goal of this study.

Our research found no differences in demographic parameters between the two groups except for body mass index. Along with our results Baikpour et al. (13) assessed demographic and anthropometric results where there was no difference between the included groups, also Erdogmus $\boldsymbol{e t}$ al. (14) reported similar data and for BMI there was a difference between the participants.

There were no statistically significant differences between the study groups in terms of the basal Laboratory findings. Along with our results Baikpour et al. ${ }^{(13)}$ reported that there was no difference between the included groups, also Erdogmus et al. ${ }^{(14)}$ reported similar data.
Doppler flow characteristics differed significantly between the two groups, also. For all other Doppler flow characteristics, except for the minimum velocity (Vmin), there was no difference between the two groups according to Balasubramanian et al. ${ }^{(16)}$.

AUC corrected for VPI optimism was considerably higher than AUC corrected for BARD scoring, but not for NAFLD FS, APRI, or FIB-4 scoring. As a result of including VPI in all clinical risk models, the optimism-corrected AUC grew statistically significantly. The only risk model that increased corrected AUC statistically significantly when combined with VPI was FIB-4. We ran a sensitivity analysis in a subset of patients who had a duplex Doppler ultrasonography within six months of a liver biopsy to see how sensitive our findings were to the time between sonography and biopsy. According to our findings, VPI levels were lower in patients with advanced liver fibrosis. According to other studies using shear-wave elastography and serum biomarkers, prediction based on VPI performs similarly like Baikpour et al. ${ }^{(13)}$ and Samir et al. ${ }^{(17)}$. All previous clinical prediction models improved their diagnostic performance significantly when VPI was included. VPI appears to measure a previously 
undetected sonographic marker of moderate or greater liver fibrosis in NAFLD patients, according to the study's conclusions. These results agree with Baikpour et al. ${ }^{(13)}$. It has previously been suggested that alterations in the portal vein's sonography, such as the inversion or diminution of antegrade flow volume, could indicate liver illness ${ }^{(\mathbf{1 8})}$. Alterations in portal vein pulsatility began to be studied in the early 1990s. ${ }^{(19)}$. During peak systole, venous congestion of the hepatic parenchyma within the liver capsule leads to competition between portal vein and hepatic artery inflow, increasing portal vein pulsatility, according to Westra et al. ${ }^{(19)}$. In Erdogmus et al. ${ }^{(14)}$ study, they reported decreased VPI in NAFLD patients compared to healthy controls. They concluded that this change was caused by impaired liver vascular compliance as a result of lipid infiltration. Similar findings are found by Balci $\boldsymbol{e t}$ al. ${ }^{(20)}$ in research involving 105 patients with NAFLD and 35 healthy controls. 90 NAFLD patients and 90 healthy control subjects were evaluated using the VPI score and the same conclusion was reached as in previous studies, which were conducted by Balasubramanian $\boldsymbol{e t}$ al. (16) Another study's findings revealed that patients with cirrhosis have higher levels of VPI, as described by Iranpour et al. ${ }^{(11)}$. The increased resistance of hepatic venules, arterioportal diversion due to liver anatomical distortions, and the reversed flow of portal veins could all contribute to this. It is possible that impaired vascular compliance due to fat accumulation within hepatocytes was the cause of lower VPI and MFV in patients with NAFLD than in the control group in our study. We discovered no correlation between MFV or VPI scores and sonographic fatty liver assessment. This inquiry all lead to the same conclusion.

As a result, ultrasonography cannot reveal the existence or absence of MFV/VPI/hepatic vein flow abnormalities such as fibrosis, necrosis, or inflammation, and hence liver biopsy is required to determine whether or not these conditions exist. As a result, these restrictions may account for the variations in the impact of fatty liver severity on the VPI, hepatic vein spectrum and MFV.

Along with our results, Patients with fatty liver had slower portal flow (Vmax and MFV) and lower portal VPI than healthy controls, according to Balasubramanian et al. ${ }^{(16)}$ and this was associated with the degree of fatty liver. Even though the association was weak, only HARI (r-value of -0.517) and VPI ( $r$-value of -0.449) were more closely linked to the severity of NAFLD than Vmax (r-value of 0.293 ) and MFV (r-value of -0.182) in terms of NAFLD grading.

There were statistically significant differences between the case and control groups in all Doppler indicators that combined with our results in Vmin, as well as Vmax where Vmin, and VPI in the case group were lower than in the control group with respect to portal vein Doppler indices ${ }^{(21)}$. According to these data, liver infiltration with fat raises flow resistance in the portal vein, resulting in reduced hepatic portal blood flow, which can be explained by the theory. Hepatic artery Doppler study by Sabry et al. (21) found that HARI was considerably lower in the case group than in the control group.

\section{CONCLUSION}

For people at high risk of NAFLD, we found that VPI may be a useful biomarker, especially when combined with other clinically validated prediction tools. Further research into the efficacy of VPI for the diagnosis of high-risk NAFLD is essential because standard diagnostic ultrasonography is normally included at no additional cost. Noninvasive high-risk NAFLD diagnosis may benefit from the VPI's low cost and noninvasive validation.

\section{Financial support and sponsorship: Nil. Conflict of interest: Nil.}

\section{REFERENCES:।}

1. Chalasani N, Younossi Z, Lavine J et al. (2018): The diagnosis and management of nonalcoholic fatty liver disease: practice guidance from the American Association for the Study of Liver Diseases. Hepatology, 67: 328-357.

2. Younossi Z, Koenig A, Abdelatif et al. (2016): Global epidemiology of nonalcoholic fatty liver disease - meta-analytic assessment of prevalence, incidence, and outcomes. Hepatology, 64: 73-84.

3. Machado M, Marques-Vidal P, Cortez-Pinto $\mathbf{H}$ (2006): Hepatic histology in obese patients undergoing bariatric surgery. J Hepatol., 45: 600-606.

4. Ahmed M, Barakat S, Almobarak A (2012): Nonalcoholic fatty liver disease and cardiovascular disease: has the time come for cardiologists to be hepatologists? J Obes., 12: 48-52.

5. Kleiner D, Brunt E, Van Natta $M$ et al. (2005): Non- alcoholic Steatohepatitis Clinical Research Network. Design and validation of a histological scoring system for nonalcoholic fatty liver disease. Hepatology, 41: 1313-1321.

6. Ratziu V, Charlotte F, Heurtier A et al. (2005): Sampling variability of liver biopsy in nonalcoholic fatty liver disease. Gastroenterology, 128: 18981906.

7. Mohamed A, Gohary K, Mashad G et al. (2016): Interleukin 10, thyroid status and ferritin are noninvasive prognostic biomarkers for diagnosis of fatty liver disease in children. Journal of International Research in Medical and Pharmaceutical Sciences, 8: 85-93.

8. Gawrieh S, Chalasani N (2013): NAFLD fibrosis score: is it ready for wider use in clinical practice and for clinical trials? Gastroenterology, 145: 717-719.

9. Castéra L (2009): Transient elastography and other noninvasive tests to assess hepatic fibrosis in patients with viral hepatitis. J Viral Hepat., 16: 300-14.

10. Tovo $C$, Villela-Nogueira $C$, Leite $N$ et al. (2019): Transient hepatic elastography has the best performance to evaluate liver fibrosis in non-alcoholic 
fatty liver disease (NAFLD). Ann Hepatol., 18: 445449.

11. Iranpour $P$, Lall $C$, Houshyar $R$ et al. (2016): Altered Doppler flow patterns in cirrhosis patients: an overview. Ultrasonography, 35: 3-12.

12. Drescher H, Weiskirchen $S$, Weiskirchen $R$ (2019): Current Status in Testing for Nonalcoholic Fatty Liver Disease (NAFLD) and Nonalcoholic Steatohepatitis (NASH). Cells, 8 (8): 845-49.

13. Baikpour M, Ozturk A, Dhyani M et al. (2020): Portal Venous Pulsatility Index: A Novel Biomarker for Diagnosis of High-Risk Nonalcoholic Fatty Liver Disease. AJR Am J Roentgenol., 214 (4): 786-791

14. Erdogmus B, Tamer A, Buyukkaya R et al. (2008): Portal vein hemodynamics in patients with nonalcoholic fatty liver disease. Tohoku J Exp Med., 215 (1): 89-93.

15. Solhjoo E, Mansour-Ghanaei F, MoulaeiLangorudi R et al. (2011): Comparison of portal vein Doppler indices and hepatic vein Doppler waveform in patients with nonalcoholic fatty liver disease with healthy control. Hepat Mon., 11: 740-744.

16. Balasubramanian $P$, Boopathy $V$, Govindasamy $E$ et al. (2016): Assessment of Portal Venous and
Hepatic Artery Haemodynamic Variation in NonAlcoholic Fatty Liver Disease (NAFLD) Patients. J Clin Diagn Res., 10 (8): 7-10.

17. Samir A, Dhyani M, Vij A et al. (2015): Shear-wave elastography for the estimation of liver fibrosis in chronic liver disease: determining accuracy and ideal site for measurement. Radiology, 274 (3): 888-96.

18. Robinson $\mathrm{K}$, Middleton W, Al-Sukaiti $\mathrm{R}$ et al. (2009): Doppler sonography of portal hypertension. Ultrasound Q., 25 (1): 3-13.

19. Westra S, Zaninovic A, Vargas J et al. (1995): The value of portal vein pulsatility on duplex sonograms as a sign of portal hypertension in children with liver disease. AJR., 165: 167-172.

20. Balci A, Karazincir S, Sumbas H et al. (2008): Effects of diffuse fatty infiltration of the liver on portal vein flow hemodynamics. J Clin Ultrasound, 36: 134-140.

21. Sabry M, Youssef T, Shaker M et al. (2021): Portal venous and hepatic artery hemodynamic variation in non-alcoholic fatty liver disease. Egypt Liver Journal, 11: 58-63. 